\title{
Screening of Probiotics Efficiency to Inhibit Persistent Biofilm-Related Infections
}

\author{
Aziz Fatima*, Kainat Ahmed Khan, Faiza Ahad \\ Department of Microbiology, Jinnah University for Women, Karachi, Pakistan.
}

\begin{abstract}
Probiotics are live bacteria. They are administered in food, with beneficial effects to the host, help to prevent or treat diseases, the majority of which are gastrointestinal. The isolation and utilization of Lactobacillus spp. as probiotics from dairy or some other items has been exhibited to be health promoting. Modification in Gut flora contribute in different sorts of disease conditions, and distinctive Microorganisms developing in a biofilm are related with constant and chronic human diseases which are resistant to antimicrobial agents. The biofilm producing organisms are E.coli, Pseudomonas, Klebsiella, Staphylococcus and many more.
\end{abstract}

Objective: The aim of study is to screen the efficiency of probiotics in the inhibition of bacterial biofilm.

Methodology: Two methods used for screening of biofilm production are, Tube method and Congo red method. Three species of Lactobacillus were isolated and screened for biofilm inhibition. Four commercial isolates of E. coli were obtained and among those, three were efficiently able to produce biofilm. All species of Lactobacillus were able to counteract the biofilm production.

Results: Lactobacillus species were identified to be L. plantarum, L. casei and L. bulgaricus on the basis of sugar test and microscopy, which inhibited the biofilm formation of intestinal bacteria.

Conclusion: After this study there is no doubt in believing that the Probiotics are definitely new hope towards treatment of infections that are prevailed among people. Conventional dairy items are great source of bacterial strains and bioactive compound discharged by these three identified strains could be utilized in pharmaceuticals for recovery from bacterial diseases.

Keywords: Infectious agents, Biofilms, Probiotics, Gastrointestinal infection, Urinary tract infections, Diarrheal diseases.

\section{INTRODUCTION}

Gram negative microorganisms can cause numerous kinds of infections and diseases and spread to people in an assortment of ways. A few categories, including $E$. coli, causative agent for food borne illness. $V$. cholera the microbes in charge of cholera is a waterborne pathogen. They can act as respiratory tract contaminants, for example, specific sorts of pneumonia and explicitly transmitted sicknesses, and urinary tract infections like gonorrhea [1].

E. coli is a Gram-negative rod shaped enteric bacterium, dominating species among all the facultative anaerobes of the gastrointestinal tract i.e. dominant flora possessing the human colonic area. Their species are generally classified by their virulence properties, systems of pathogenicity, clinical disorders, and $\mathrm{O}$ and $\mathrm{H}$ serotypes [1]. Although most species are harmless to the intestinal lumen, some procured harmfulness factors and can cause a wide scope of human diseases [2].

The pathogenic E.coli is causative agent of three clinical syndromes: Urinary Tract Infections, Enteric/Diarrheal Diseases, and Meningitis [3].

The key instruments by which E.coli cause enteric infec-

*Address correspondence to this author at the Department of Microbiology, Jinnah University for Women, Karachi, Pakistan.

E-mail: azizfatima1988@gmail.com tions incorporate connection and colonization of the intestinal mucosa, control of the host cell cytoskeleton or dodging host insusceptible protections, and generation of toxins [4]. E. coli is worldwide wellbeing load forced by diarrhea. Current interventions to inactivate out pathogenic $E$. coli include the utilization of anti-toxins. In any case, numerous pathogenic strains that can cause disease have turned out to be impervious to anti-infection agents [5]. The ascent of anti-microbial opposition has lead specialists to discover antimicrobial options of which probiotics have picked up a developing interest.

\section{SYMPTOMS OF SEVERE GASTROINTESTINAL INFECTION}

Abdominal pain, extreme stomach cramping, regularly beginning all of a sudden, watery stool and looseness of the bowels, starting a couple of hours after the torment starts, bright red wicked stools around a day coming about because of the poison damage to the digestive organs, nausea, vomiting, fatigue coming about because of dehydration [5].

With more than 250 serotypes, E. coli is an exceptionally adaptable bacterium that range from harmless gut commensal to intra or additional intestinal pathogens, including normal colonizers of medicinal gadgets and the essential drivers of urogenital infections [5]. Microbes' can develop attachment to 
pretty much every surface, framing architecturally complex networks named biofilm. Although current information of bacterial science owes a lot to work done on planktonic societies of research center strains of $E$. coli, many isolates likewise have the ability to shape biofilm structures in vivo and in vitro [6].

Bacterial cell shows two ways of growing mode for instance planktonic cell and sessile absolute which is known as the biofilm. Biofilm is a relationship of small organisms in which cells hold fast to each other on a surface encased inside system of extracellular polymeric substance released by organisms themselves [7]. A Dutch specialist, Antoni van Leeuwenhoek, suddenly watched 'animalcule' on surfaces of teeth by using a clear amplifying focal point and this was considered as the microbial biofilm disclosure. Later in 1973, Characklis exhibited that biofilms are consistent just as show higher safe nature to disinfectants for instance chlorine. Costerton, in 1978, generated the term Biofilm \& alert the world about the criticalness of biofilm.

The design of biofilm contains two central parts for instance water channel for enhancements transport and a territory of thickly squeezed cells having no detectable pores in it [8]. Biofilm improvement has following fundamental advances: Association at first to a surface, improvement of scaled down scale colony, three-dimensional structure improvement and biofilm improvement, development and dispersal [9].

\section{BIOFILM FORMATION BY E. COLI}

$E$. coli is one of numerous microscopic organisms that can switch between planktonic frame and biofilm shape. It is a rod shaped; gram-negative microorganism leading to a substantial number of hospitals acquired network contaminations, for example, urinary tract infections (UTIs) and prostatitis. It can form toxins, polysaccharide and biofilm. It can likewise in vitro form biofilm [10]. E. coli containers are increased atomic weight particles which are stick to cell surface. E.coli capsule plays indirect role by securing the adhesion of bacterial surface. Distinctive ecological conditions influence $E$. coli capacity so that to frame biofilm [11]. E. coli biofilm thickness might be of several microns \& representing a trouble in treatment with anti-infection agents because of exopolymers presence. A few reasons can clarify the requirement for microscopic organisms to make biofilm, along these lines microorganisms tolerate washing away by water streams or biofilm cells are around expectedly more safe as compare to planktonic. E. coli O157:H7 has demonstrated overall capacity to append formation of colony and frame biofilms on an assortment of surfaces [12].

\section{PROBIOTICS AS LIVING DRUGS}

Biofilm arrangement is a major determinant factor being developed of bacterial contaminations. What's more, microbes installed in a biofilm are progressively impervious to antimicrobials and in this manner the capacity of microscopic organisms to hold on and develop in a biofilm is by all accounts the central point for pathogenesis and helpful disappointment. Anti-biofilm capability of the Lactobacillus spp. standing in against to multiple drug obstruction $P$. aerugino$s a, S$. aureus and $E$. coli has been concentrated for quite a while. Conventional dairy items are felt as great source of lactic microscopic organisms (LAB) and perfect vehicles to convey advantageous microorganisms to human gut tract [13]. LAB is extraordinary probability to be used as probiotics due to their nonpathogenic nature, incredible antimicrobial development and security from gastrointestinal condition. Multi drug resistant creatures are incorporated with a couple of system picked up and nosocomial pollutions similarly as foodborne infections. Biofilm plan is seen as a noteworthy determinant factor being created of pollution by pathogenic microscopic organisms [14].

\section{THE THERAPEUTIC USE OF PROBIOTICS}

Probiotics are live microscopic life forms and yeasts that are useful for you, especially your stomach related system. We generally consider these as germs that reason disorders. Regardless, your body is stacked with organisms, both extraordinary and awful. Probiotics are routinely called "incredible" or "pleasing" microorganisms since they help keep your gut sound. The term of probiotic is gotten from Greek word meaning life. According to the latest report of the Food and Agriculture Organization (FAO) and World Health Organization (WHO), probiotics are live microorganisms whose attractive affirmation causes important effects on wellbeing [15]. These microscopic organisms cause decrease of gastrointestinal sicknesses by expanding advantage microorganisms' development and lessening pathogens' population mechanisms. Positive LAB is broadly disseminated in the condition that can keep the development of pathogenic microorganisms by creating specific substances [16]. According to scientific reports, anti-allergic and anticancer impacts, expanding fat misfortune and insusceptible reaction of the host, improvement symptoms of bowel syndrome, intestinal inflammation, and anti-toxin prompted looseness of the bowels are other valuable impacts of probiotics. Yogurt is being seen as a sort of healthy nourishment which has a low fat and fundamental value as far as protein and nutrients. Previously, yogurt was made at home by basically enabling milk to mature. As of late, most yogurts are made by utilizing extraordinary lactic corrosive delivering microbes. Common place generation of yogurt includes a symbiotic relationship of the starter cultures Lactobacillus bulgaricus and Streptococcus thermophilus, in a 1:1 ratio [17].

\section{STUDY AIM}

To isolate the probiotics from natural products and determine high efficacy of probiotics against bacterial biofilm. 


\section{MATERIALS AND METHODOLOGY}

Three different samples were used for the isolation of Probiotics from the dairy products, which includes: Milk, Yogurt and Cheese.

Each sample was weighed up to $10 \mathrm{gm}$. as per requirement \& transferred in separate test tubes. Now $1.5 \mathrm{ml}$ of PBS was transferred in each tube and the tubes were vortex for 1-2 min. Test tubes containing $4 \mathrm{ml}$ of MRS broth and transferred $1 \mathrm{ml}$ from each sample tube into the broth to enrich the samples for growth enhancement and incubated for 24 hours at $37^{\circ} \mathrm{C}$.

Next day used De Man Rogosa and Sharpe (MRS) agar, streaking was done on plates and incubated for 24 hours at $37^{\circ} \mathrm{C}$ for the growth of probiotics. Next day after 24 hours colonies were showed on MRS agar and microscopy were done by gram staining. After that some catalase test and sugar tests were applied like lactose, mannitol, sucrose, glucose for the confirmation of microbial specie of probiotics, and then antimicrobial activity of probiotics was checked.

\section{ISOLATES OF E. COLI WERE USED TO DETECT BIOFILM PRODUCTION}

\section{Tube Method}

Biofilm Production: Trypticase soy broths with 1\% glucose were used for this test. Take loop full of organism (all 4 strains) and inoculate each in $10 \mathrm{ml}$ in 4 different tubes. Incubate the tubes for 24 hours at $37^{\circ} \mathrm{C}$.Discard medium gently next day. Washed with phosphate buffer saline PBS (ph 7.3) and let it dry. After drying, stain the tubes with $1 \%$ Crystal Violet. Excess stain is washed off with deionized water. Leave tubes to air dried again in inverted position. Visible circular line in the center and bottom of test tube are the indication of positive result. Absence of line; result are considered as negative.

\section{Congo Red Agar Method}

Biofilm Production: Congo red agar (CRA) prepared with brain heart infusion (BHI) broth, agar in $10 \mathrm{~g} / \mathrm{L}$, Congo red indicator were used with the concentration of $8 \mathrm{~g} / \mathrm{L}$. After preparation of Congo red stain in concentrated aqueous solution, autoclave $\mathrm{BHI}$ agar with sucrose at $55^{\circ} \mathrm{C}$. CRA were poured in 4 plates with test organisms by lawing method under sterilized condition. Plates were then incubated at $37 \mathrm{oC}$ for 24 hours. Black, dark colonies with dry consistency indicate biofilm production.

Biofilm Inhibition: After confirmation of biofilm production, followed by inhibition test. Lawn of 3 test strains of $E$. coli were made in CRA plates (These three strains were able to produce biofilm except one). Now make one-line streak of $L$. bulgaricus, L. casei and L. plantarum on all the plates. The plates were incubated for 24 hours at $37^{\circ} \mathrm{C}$. Results were observed after 24 hrs.

\section{Well Plate Method}

Biofilm Production: Take a loop full of culture from each test strains and inoculate in 4 different tubes of tryptic soy (TS) broth. In 96 wells plate, $100 \mu$ from each tube pour in well (make sure to label it properly). For confirmation of results 4-8 replicate were performed. Incubate the plate for $24 \mathrm{hrs}$ at $37^{\circ} \mathrm{C}$. Biofilms of the pathogenic bacteria form in micro-titer plates within $24 \mathrm{hrs}$. Then, the medium was discarded from each well. 50 $\mu$ L PBS was used for washing. Biofilm production was observed at the bottom of wells.

Biofilm Inhibition: Different suspension consisting of Lactobaccilus strains were then poured in each well about $100 \mu \mathrm{L}$. The plates were incubated for a further $24 \mathrm{~h}$ at $37^{\circ} \mathrm{C}$. After incubation, the medium was discarded. Wash the wells with PBSD is card it and then wash with deionized water. Now last washing is done using Crystal violet. Afterwards discarded and observe weather the biofilm production is inhibited by the Lactobacillus.

\section{RESULTS}

Using different samples from natural products, species of lactobacillus were isolated and identified using gram staining as shown in Fig. (1) and biochemical characterization was done using Sugar fermentation test as mentioned in Table $\mathbf{1}$.

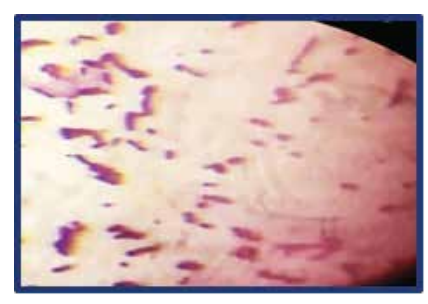

L.casei

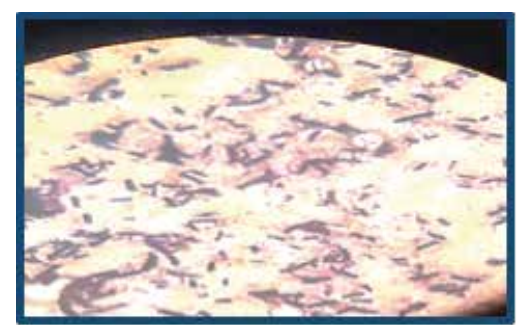

L. bulgaris

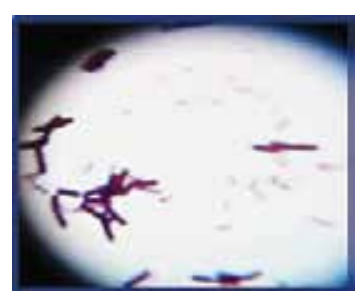

L. plantarum
Fig. (1). Species of Lactobacillus isolated from different dairy products.

Table 1. Sugar Fermentation Test.

\begin{tabular}{|l|c|c|c|}
\hline Sugar Tubes & Milk & Cheese & Yogurt \\
\hline Sucrose & $+\mathrm{ve}$ & $+\mathrm{ve}$ & $-\mathrm{ve}$ \\
\hline Glucose & $+\mathrm{ve}$ & $+\mathrm{ve}$ & $-\mathrm{ve}$ \\
\hline Lactose & $+\mathrm{ve}$ & $+\mathrm{ve}$ & $+\mathrm{ve}$ \\
\hline Mannitol & $+\mathrm{ve}$ & $+\mathrm{ve}$ & $+\mathrm{ve}$ \\
\hline
\end{tabular}




\section{TUBE METHOD}

Four commercial isolates of E.coli were used to detect biofilm production. Biofilm production. Was clarified using crystal violet Tube method as shown in Fig. (2) and Congo red method as shown in Fig. (3).

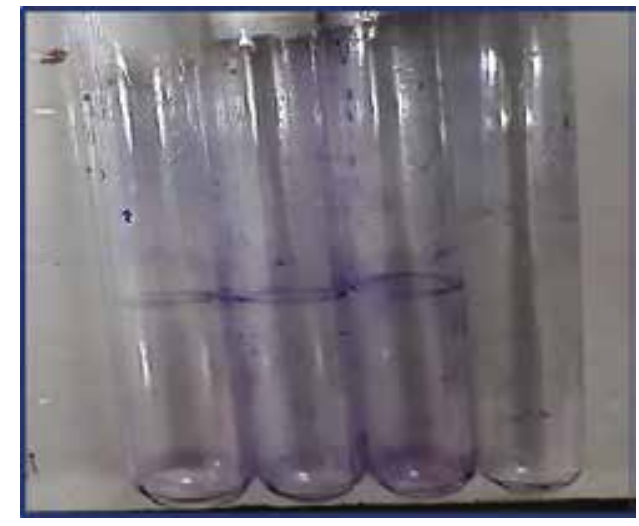

Fig. (2). Biofilm Appeared in Three Tubes, Indicating that Three Strains of E. Coli.

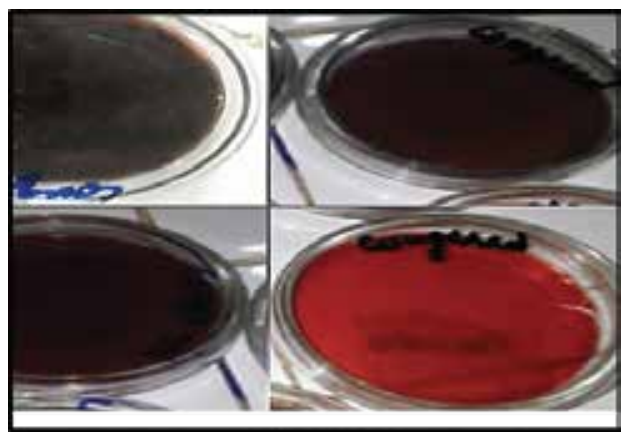

Fig. (3). Screening of Biofilm using Congo Red.

Inhibition at different \% was observed on the plates. The black color indicates the growth of test organism and the region indicates the inhibition as shown in Fig. (4).

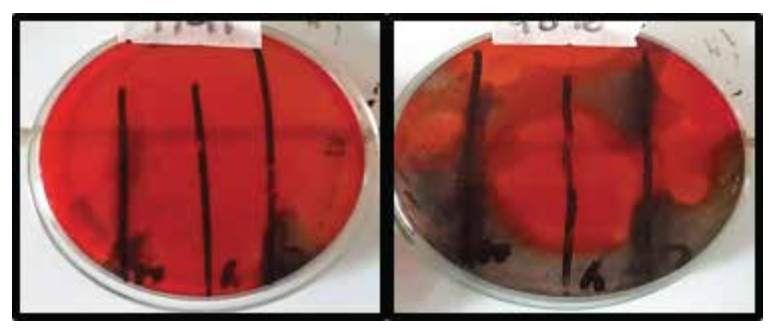

Fig. (4). Inhibition of Biofilm using Lactobacillus Screened by Congo Red Agar Method.

\section{DISCUSSION}

For a long time, dairy items have been perceived as important items to human wellbeing. As of late, numerous researchers have separated and recognized $\mathrm{LAB}$ and lactobacilli from conventional items worldwide and have assessed their impacts against different pathogens. Microorganisms, for example, lactobacilli and numerous other microscopic organisms can take out pathogens through various instruments [17]. Their impact of probiotics against $E$. coli was one of the achievements since it was prevailing with regards to restraining the development of the biofilm in each one of those three strains.

A few examinations have been directed on creatures which demonstrate that probiotics have anti-tumor property just as cell reinforcement property to battle contaminations. Probiotics have important capacities to diminish stomach related agony, helps in sensitive bowel, ulcerative colitis and is valuable to stay away from hypersensitivities $[17,18]$. Probiotics have a rundown of instrument of activity extending from immunomodulation, short chain unsaturated fat, bacteriocin creation, bringing down of gut $\mathrm{pH}$ and supplement rivalry to stimulate obstruction capacity of mucosa [18]. Antagonistic activity of three isolated lactobacilli of some dairy items was assessed in this investigation. Further, test uncovered that the species of lactobacillus was $L$. plantarum, $L$. casei and, $L$. bulgaricus. The acquired outcomes demonstrated that every one of the three separates had moderate movement aside from $L$. plantarum that had generally solid movement against $E$. coli separately. Antimicrobial impact of Lactobacilli against pathogens is chiefly because of the generation of natural acids and $\mathrm{pH}$ decrease in co culture with pathogenic microscopic organisms in spite of the fact that they can deliver some different substances [19].

Antimicrobial movement of $L$. bulgaricus strain which were isolated from homemade yogurt sample was watched. E. coli biofilm generation is one of the genuine concerns which make it progressively confounded. Henceforth, the reason for this whole research was to get mindful of the way that probiotics are one of the primary weapons to fix and manage living beings that are biofilm makers. Nowadays, different common items are utilized to treat sicknesses. In light of the consequences of this examination, present probiotic microorganisms in characteristic assets can be utilized for restraint and decrease of pathogens [20].

\section{CONCLUSION}

After this entire study there is no uncertainty in trusting that the Probiotics are unquestionably new expectation towards infections that are won among individuals, including enteric pathogens and antibacterial impacts of their metabolites are dynamic and stable under various states of temperature and sharpness. This work showed that conventional dairy things are incredible wellsprings of valuable bacterial strains and bioactive compound released by these strains could be used in pharmaceutical organization against bacterial infections and contamination. This entire test will be assessed here in light of the latest outcomes got in this research. 


\section{CONFLICT OF INTEREST}

Declared none.

\section{ACKNOWLEDGEMENTS}

Declared none.

\section{REFERENCES}

[1] Montville TJ, Matthews KR. Food Microbiology: An Introduction. Washington (DC): ASM Press 2005; p. 380.

[2] Nataro JP, Steiner T, Guerrant RL. Enteroaggregative escherichia coli. Emerg Infect Dis 1998; 4(2): 251-61. DOI: $10.3201 /$ eid0402.980212

[3] Kaper JB, Nataro JP, Harry LTM, Mobley HL. Pathogenic escherichia coli. Nat Rev Microbiol 2004 ; 2: 123.

DOI: $10.1038 /$ nrmicro 818

[4] Torres AG. Intestinal pathogenic escherichia coli. In: Barrett ADT, Stanberry LR, Eds. Vaccines for Biodefense and Emerging and Neglected Diseases. USA: Academic Press 2009; pp. 1013-29.

DOI: 10.1016/B978-0-12-369408-9.00051-2

[5] Collignon P. Resistant escherichia coli --we are what we eat. Clin Infect Dis 2009; 49: 202-4. DOI: 10.1086/599831

[6] Besser RE, Griffin PM, Slutsker L. Escherichia coli O157: H7 gastroenteritis and the hemolytic uremic syndrome: An emerging infectious disease Annu Rev Med 1999; 50: 355-67.

DOI: 10.1146/annurev.med.50.1.355

[7] Hall-Stoodley L, Costerton JW, Stoodley P. Bacterial biofilms: From the natural environment to infectious diseases. Nat Rev Microbiol 2004; 2: 95-108.

DOI: $10.1038 /$ nrmicro 821

[8] Costerton J, Stewart PS, Greenberg EP. Bacterial biofilms: A common cause of persistent infections. Science 1999; 284: 1318-22. DOI: 10.1126/science.284.5418.1318

[9] Donlan RM, Costerton JW. Biofilms: Survival mechanisms of clinically relevant microorganisms. Clin Microbiol Rev 2002; 15: 167-93. DOI: 10.1128/CMR.15.2.167-193.2002

[10] Naves P, del Prado G, Huelves L. Effects of human serum albumin, ibuprofen and $\mathrm{N}$-acetyl-1-cysteine against biofilm formation by pathogenic Escherichia coli strains. J Hosp Infect 2010; 76: 165-70. DOI: 10.1016/j.jhin.2010.05.011
[11] Larson EL, Gomez-Duarte C, Lee LV, Della-Latta P, Kain DJ, Keswick BH. Microbial flora of hands of homemakers. Am J Infect Control 2003; 31: 72-9. DOI: 10.1067/mic.2003.33

[12] Uhlich GA, Cooke PH, Solomon EB. Analysis of the red-dry-rough phenotype of an escherichia coli O157: H7 strain and its role in biofilm formation and resistance to antibacterial agents. Appl Environ Microbiol 2006; 72: 2564-72. DOI: 10.1128/AEM.72.4.2564-2572.2006

[13] Zamani H. Isolation of a potentially probiotic Lactobacillus plantarum from Siahmezgi cheese and its characterization as a potentially probiotic. Biol J Micro Organ 2016; 4: 97-108.

[14] Salehzadeh A, Zamani H, Langeroudi MK, Mirzaie A. Molecular typing of nosocomial Staphylococcus aureus strains associated to biofilm based on the coagulase and protein a gene polymorphisms. Iran J Basic Med Sci 2016; 19: 1325-30.

[15] Wouters JTM, Ayad EHE, Hugenholtz J, Smit G. Microbes from raw milk for fermented dairy products. Int Dairy J 2002; 12: 91-109. DOI: 10.1016/S0958-6946(01)00151-0

[16] Shokryazdan P, Sieo CC, Kalavathy R, et al. Probiotic potential of Lactobacillus strains with antimicrobial activity against some human pathogenic strains. BioMed Res Int 2014; 2014: 927268. DOI: $10.1155 / 2014 / 927268$

[17] Kim Y, Kim SH. Released exopolysaccharide (r-EPS) produced from probiotic bacteria reduce biofilm formation of enterohemorrhagic Escherichia coli O157: H7. Biochem Biophys Res Commun 2009; 379: 324-9.

DOI: $10.1016 /$ j.bbrc.2008.12.053

[18] Fatima A. Probiotics as living drugs to resolve urinary tract infections. Nat J Health Sci 2017; 2: 144-9. DOI: 10.21089/njhs. 24.0144

[19] Milioni C, Martínez B, Degl'Innocenti S, et al. A novel bacteriocin produced by Lactobacillus plantarum LpU4 as a valuable candidate for bio preservation in artisanal raw milk cheese. Dairy Sci Tech 2015; 95: 479-94.

DOI: $10.1007 / \mathrm{s} 13594-015-0230-9$

[20] Barrons R, Tassone D. Use of Lactobacillus probiotics for bacterial genitourinary infections in women: A review. Clin Ther 2008; 30(3): 453-68. DOI: 10.1016/j.clinthera.2008.03.013 